\title{
COST EFFECTIVE IN-HOUSE PRODUCTION PROCESSES AND EVALUATION OF CEPHALOSPORINS AND METFORMIN
}

\author{
Mohd Riyaz Beg ${ }^{1}$, Faith Dias ${ }^{2}$, Kashish Garg ${ }^{3}$, Vanshika Yadav ${ }^{4}$, Kshitiz Luthra ${ }^{5}$ \\ ${ }^{1}$ Institute of Chemical Technology, Mumbai, Maharashtra, India \\ ${ }^{2}$ Indian Institute of Technology, Bombay, Mumbai, Maharashtra, India \\ ${ }^{3}$ Amity University, Noida, UP, India \\ ${ }^{4}$ SGT University, New Delhi, Delhi, India \\ ${ }^{5}$ Delhi Institute of Pharmaceutical Sciences and Research, New Delhi, Delhi, India
}

\section{Corresponding Author: Mohd Riyaz Beg}

Address: Dept. of Pharmaceutical Sciences and Technology, Institute of Chemical Technology, Mumbai-400019, Maharashtra, India

Email: mohdriyazbeg@gmail.com

\begin{abstract}
The report deals with understanding the problems that have arisen in the Pharmaceutical industry and the classes of drugs which are suffering the brunt of Chinese influx in the Indian market. Since our thrust area itself has to deal with the problems being faced by the pharmaceutical industry and the lack of self- reliance when it comes to APIs, we first decided to go with official government data of the APIs being imported from China and work commenced from there. Based on government data, market share, consumption, pricing of drugs and import reports, we selected two drugs, one being an anti-bacterial drug which requires fermentation namely, cephalosporins and the other being a well-known anti-diabetic drug, Metformin. Currently, we are looking at the various technologies being used in producing the two drugs which were shortlisted and, we'll be further finding out the loopholes in the system and fixing it or suggesting novel techniques to make the Indian Pharmaceutical industry a powerhouse. Both cephalosporins and metformin have been majorly imported from China due to several loopholes in producing them here in India and to add to it, the strategic pricing employed by the Chinese manufacturers gives them an edge over indigenous products. The manufacturing processes and regulations for both cephalosporins and metformin, need to undergo an overhaul in order to compete with giants like China and thus displace them from their position of number 1 in this field. Finding loopholes in the technology and regulatory factors, suggesting novel technologies or improvements within current processes and performing a detailed cost analysis.
\end{abstract}

Key words: Cost effective, Technology, APIs, Indian Pharmaceutical Industry 


\section{Introduction}

India faced major problems when COVID-19 hit the whole world and the import- export business was largely affected due to lockdown imposed by different countries and India itself. India although being one of the largest pharmaceutical industries in the world lacks sophisticated API producing technology which are cost effective. The below mention points will state the problems with our pharmaceutical industries:

\subsection{Pricing Pressures:}

The increased rate of wholesale consolidation in the US market led to considerable decline in the bargaining power of exporting countries, and has especially impacted the Indian pharmaceutical industry leading to pricing pressures. During the tenure of 2016, 3 players in the US pharma distribution market, viz. AmerisourceBergen Corp, Cardinal Health Inc. and McKesson Corp, together held about $85 \%$ of the market share and estimated to have reached US\$ 424 billion in 2017, a $4.5 \%$ increase from the 2016 estimate.

\subsection{Excessive Dependence on China for Drug Imports}

India is heavily dependent on China for bulk drug intermediates and APIs with the country accounting for nearly two-third of India's imports of such products. An over dependence on bulk drugs, active pharmaceutical ingredients and other raw materials from China, had an unfavourable impact on the Indian pharmaceutical industry in 2020. Discontinuance of supply triggered a major loss for the pharma players. During the year 2016-17, China had a share of $66.7 \%$ in the aggregate bulk drug imports by India, valued at US\$ 1.83 billion. Recorded quantity of imports, China contributed about 60.7 percent of the total bulk drug imports. The Chinese market's competitive advantage lies in the ability to provide low-cost raw materials

\subsection{Infrastructural Issues}

Indian pharmaceutical industry also faces the challenge of inadequate infrastructural support such as lack of animal breeding facilities and good laboratory practices. Deficiency of skilled laboratory technicians to supervise and administer the activities and decipher the information from tests has also been not proper and largely unfavourable. Initially, huge capacity was created by both the public and private sector to cater to growing demand. However, because of the cheap rates, substantial quantities were being imported from China which forced local manufacturers to shut down operations owing to commercial unviability. As the sole manufacturer of penicillin, China has started manufacturing intermediates from penicillin $G$ (6-APA, 7-ADCA and 7-ACCA) and therefore has strategically priced penicillin, which makes even the production of intermediates uneconomical in India. 


\subsection{Technology Transfer}

There are various important discoveries which are started in the academic column. For the development of the pharmaceutical industry, it is really important to have a collaboration between academia and the industry. The transfer of technology between these two entities is really important and there should be adequate partnerships and arrangements to favour the same. In India, the earnings from technology transfer and the academia patenting rates are comparatively less.

\subsection{Stricter implementation of pollution control norms}

This is leading to higher costs of manufacturing APIs in India. Under the current norms, companies have to go through a fresh approval process every time they want to make a change in the product mix, a process that can take as long as 4 months. For increase in production or addition of equipment, it could be 8 months or even more.

\subsection{Interpretation of DPCO, 2013}

In order to cope with decisions related to scheduled v/s non-scheduled formulations, new drugs, demand notices for overcharging, etc., Indian pharma companies were forced to evolve business strategies to move up the value chain and focus on commercially attractive segments like finished formulations and complex-to-manufacture APIs. Local formulation players thus started sourcing raw materials and simple APIs from cost-competitive locations like China, which has led to increased dependence on a single source and huge fluctuations in API prices.

\subsection{No tax incentives, higher utilities and borrowing cost}

Non-availability of tax Incentives to boost API parks, higher borrowing and utilities cost (e.g., electricity, water, and steam) and low import duties have led to cheaper imports fromgiant plants in China, which enjoyed economies of scale.

\subsection{Lack of mega bulk drug parks}

Lack of large clusters for bulk drug manufacturing having common facilities for pollution control, effluent treatment and single environmental clearance leads to higher capex requirement. In India APIs such as Cephalosporins (Antibiotics) and Metformin (AntiDiabetic) are costly and their production in Indian industry is not huge. They are imported into India from other countries and formulated into desired drugs. 
Table 1: Leading Therapy Areas: Spending and Growth in Select Developed and Pharmerging Markets

\begin{tabular}{|l|l|l|l|}
\hline Therapy Area & $\begin{array}{l}2012-17 \text { CAGR constant } \\
\text { US\$ bn }\end{array}$ & $\begin{array}{l}2017-22 \text { CAGR constant } \\
\text { US\$ bn }\end{array}$ \\
\hline Diabetics & $16.9 \%$ & $8-11 \%$ & \\
\hline Antibiotics/Vaccines & $3.2 \%$ & $1-4 \%$ & \\
\hline
\end{tabular}

CAGR- Compound Annual Growth Rate.

Notes: includes 8 developed countries and 6 pharmerging countries: US, France, Germany, Italy, Spain, UK, Japan, Canada, China, Brazil, Russia, India, Turkey, Mexico Source: IQVIA Market Prognosis.

Table 2: Region Wise Global Spending of Medicines

\begin{tabular}{|l|l|l|}
\hline Region/Country & 2017 Global Spending (US\$ bn) & $\begin{array}{l}2013-17 \\
\text { US\$ }\end{array}$ \\
\hline Developed & $\mathbf{7 5 3 . 2}$ & $\mathbf{5 . 8 \%}$ \\
\hline The US & 466.6 & $7.3 \%$ \\
\hline EU 5 & 154.4 & $4.4 \%$ \\
\hline Germany & 45.1 & $4.9 \%$ \\
\hline France & 33.1 & $1.3 \%$ \\
\hline Italy & 29.0 & $5.5 \%$ \\
\hline The UK & 25.7 & $6.9 \%$ \\
\hline Spain & 21.5 & $4.6 \%$ \\
\hline Japan & 84.8 & $2.0 \%$ \\
\hline Canada & 20.7 & $3.9 \%$ \\
\hline S Korea & 13.7 & $4.5 \%$ \\
\hline Australia & 13.1 & $4.7 \%$ \\
\hline Pharmerging & $\mathbf{2 6 9 . 6}$ & $\mathbf{9 . 7 \%}$ \\
\hline China & 122.6 & $9.4 \%$ \\
\hline Brazil & 33.1 & $11.5 \%$ \\
\hline
\end{tabular}




\begin{tabular}{|c|c|c|}
\hline India & $\underline{19.3}$ & $\underline{11.0 \%}$ \\
\hline Russia & 14.9 & $10.8 \%$ \\
\hline $\begin{array}{l}\text { Other Pharmerging } \\
\text { Countries }\end{array}$ & 79.7 & $8.9 \%$ \\
\hline Rest of the World & 112.3 & $2.0 \%$ \\
\hline Total & 1135.1 & $6.2 \%$ \\
\hline
\end{tabular}

The percentage of API imports from China has spiked from around 1\% in 1991 to about 70\% in 2019 ! In the recent past, there has been a steep increase in the market price of APIs being imported from China.

Analysis based on critical APIs shows that $50 \%$ of the critical APIs are being imported and almost all the imports are from China and domestically produced APIs hold approximately $50 \%$ of the total quantity but, key starting materials for most of the APIs are still imported from China . The Chinese API industry has a boon because of economies of scale and support from the Chinese government in the form of financial incentives, infrastructure and regulatory policies such as lower capex requirements due to large Special Economic Zones (10-15 times the size of Indian SEZs), lower borrowing costs i.e. 5-7\% v/s 11-14\% in India, lower logistics costs i.e. $1 \%$ of total costs in China v/s 3\% for India and lower conversion costs as labour and electricity costs in China are relatively cheaper (an average of $11 \mathrm{US}$ cents/kw h v/s 19 US cents/kWh in India). Indian API manufacturers lost their competitive edge in the manufacture of APIs at the lower end of the spectrum and fermentation technologies (for Cephalosporins) to countries like China, largely on account of factors such as stricter implementation of pollution control norms, leading to higher costs of manufacturing APIs in India, issues in interpretation of the Drug Price Control Order (DPCO), 2013, no financial incentives like lower tax, cheaper utilities and land subsidy to lower capex requirement, lack of large-scale mega parks to manufacture bulk drugs and collapse of the fermentation industry in India.

Table 3: The APIs focused on this paper with the intermediates

\begin{tabular}{l|l|l} 
S. & Name of intermediate & Name of API synthesized \\
no. & & \\
\hline 1. & 7-ACA (Aminocephalosporanic Acid) & Cephalosporin \\
\hline
\end{tabular}


Table 4: Systemic antibiotics use in India, by antibiotic class, 2008-2012.

\begin{tabular}{|c|c|c|c|c|c|}
\hline & 2008 & & $\begin{array}{ll}2010 & 201 \\
& 1\end{array}$ & & 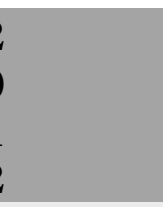 \\
\hline Systemic antibiotics & $\operatorname{DID}(\%)$ & $\operatorname{DID}(\%)$ & $\operatorname{DID}(\%)$ & $\operatorname{DID}(\%)$ & $\operatorname{DID}(\%)$ \\
\hline Quinolones & $4.0(30.7)$ & $4.1(28.8)$ & $4.2(27.70$ & $3.9(24.9)$ & $3.7(23.4)$ \\
\hline $\begin{array}{l}\text { Cephalosporins, } \\
\text { carbapenem and } \\
\text { monobactam }\end{array}$ & $1.7(12.7)$ & $2.0(14.1)$ & $2.3(15.2)$ & $2.5(16.1)$ & $2.6(16.3)$ \\
\hline
\end{tabular}

India's exports of pharmaceutical products, including drug formulations were valued at US\$ 16 billion during the year 2016-17, recording a year-on-year decline of nearly $1.2 \%$. During the seven-year period 2010-11 to 2016-17, a negative growth rate in export of these products was observed for the first time during the year 2016-17. The growth rate peaked during the period 2011-12, at 25.3\%. Post this period, a decline in growth rates was witnessed up until 2014-15 after which it increased to $9.9 \%$ in 2015-16, as the value of exports stood at US\$ 16.2 billion. However, in 2016-17, the growth actually entered the negative domain, declining by $1.2 \%$ (negative).

The import value of bulk drugs by India recorded a CAGR of $1.1 \%$ during the seven-year period between 2010-11 and 2016-17. In the year 2016-17, the value of imports stood at US\$ 2.74 billion, registering a sharp y-o-y decline of $15.7 \%$.

\section{Technological aspects involved in the manufacturing processes of cephalosporins and a general comparison of the various equipment used thereof}

Most Indian industries do not have the technological prowess and even if they do, they cannot afford to set up plants with gigantic capacities since the regulations in place do not allow for massive expansions. So, our approach has to be such that we develop technologically adept machinery which can continuously develop products at a much fasterrate with little to no losses incurred in procurement of resources and finance. Thus, developing highly robust and smooth systems in place which can outdo foreign 
propagandas of expansion and monopoly. To that a comparison of the reactors used and the extraction processes involved is given here.

\section{General steps for the production of Cephalosporin C $(\mathrm{CPC})$}

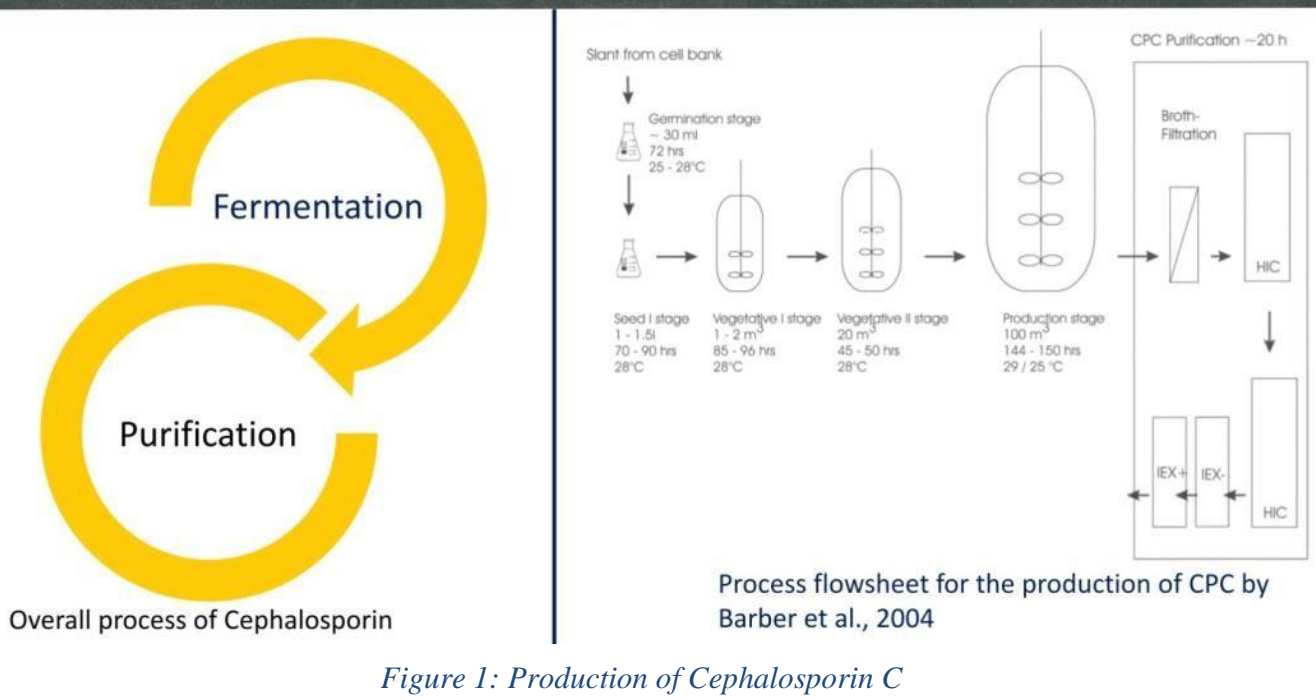

\subsection{Raw Material selection}

The selection of raw materials should be such that they fit the purpose, ensure maximum yield of the product and are easily to acquire with little to no costs.

In the case of cephalosporins, the use of lactose is replaced by sucrose and glucose which aims in reducing the costs. Invert sugar reduces the costs of procuring raw materials.

\subsection{Reactors used for the process of fermentation of Cephalosporin $\mathrm{C}$}

\section{A general classification of Bioreactors}

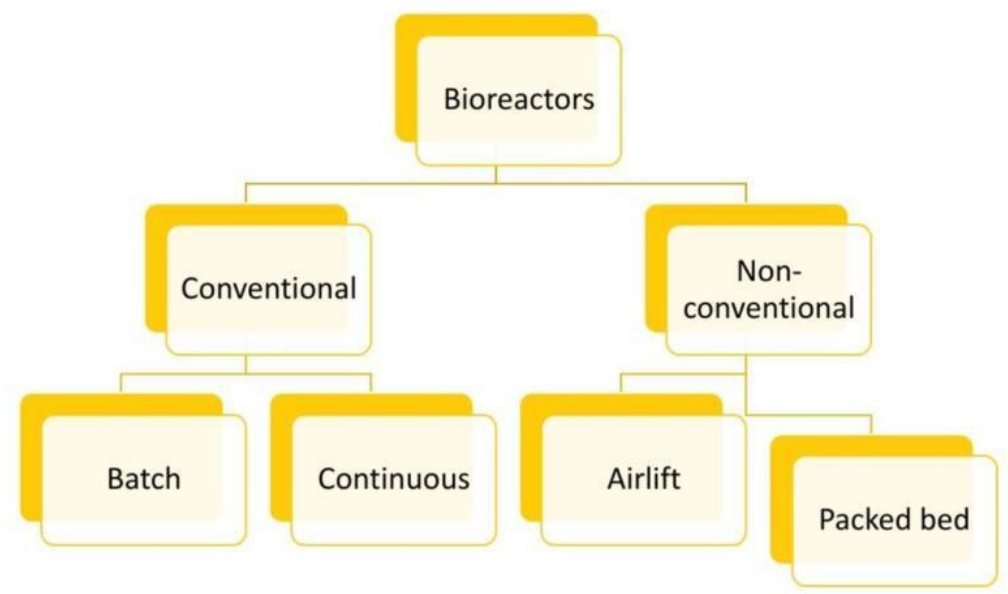


The reactors used for production of cephalosporins, are basically of 2 categories namely conventional bioreactors and non- conventional bioreactors. The conventional ones are the batch and continuous stirred tank reactors whereas, the non- conventional modes comprise of airlift bioreactors, packed bed bioreactors to name a few. Herein, we will be comparing the various bioreactors currently stated in literature and through our comparison and analysis we will be suggesting a bioreactor which will be most suited for the product and which will reduce costs drastically.

\subsubsection{Stirred tank bioreactors}

Stirred tank bioreactors are easy to scale up and the oxygen transfer ability of the reactor is good. However, stirred tank bioreactors are known for high power consumption, high shear, also the sealing and stability of the shafts present in tall bioreactors is questionable. That being said, the production of cephalosporins is done by aerobic fermentation and these reactors fulfil the purpose. However, the shear sensitive nature of the filamentous microorganisms, poses another big challenge in using these reactors. ${ }^{(1)}$

\subsubsection{Fed batch reactors}

Fed batch reactors are labor intensive, but they provide more flexibility than batch reactors. Since the rate of the substrate can be controlled maximum product of the desired production can be obtained. Formation of the desired product can be de- repressed by controlling the growth rate in a fed batch reactor. This kind of a reactor allows for the controlled feeding of nutrients. The control of the reaction rate is possible in this type of a reactor which helps to circumvent the problems faced due to oxygen transfer and cooling. ${ }^{2,4)}$

\subsubsection{Air lift bioreactors}

Airlift bioreactors are energy efficient as compared to stirred fermenters. Internal and external loop bioreactors have good production abilities since, they are gentle on fungi and actinomycetes imparting a low shear than the conventional stirred tank reactors that are in place. These reactors are operated in batch mode. The process is cost intensive and tough. Oxygenation isn't an issue with these reactors as proper oxygenation and agitation is achieved. The internal loop air lift reactors have better oxygenation and are preferred over the external loop air lift reactor. ${ }^{(3)}$

\subsubsection{Continuous stirred tank reactor}

The continuous method ends up being time consuming since the microorganisms are first 
grown in batch followed by the continuous mode. The same issues faced in batch stirred tank reactor are faced here such as high shear. ${ }^{(5)}$

\subsubsection{Packed bed bioreactor}

This reactor can be run in both batch and continuous mode along with sufficient residence time and microbial usability. Oxygen is produced in-situ in this method. ${ }^{(1)(5)}$

\subsubsection{Surface liquid culture and solid -state fermentation}

These two reactors aren't very favorable due to the high probability of lack of oxygen. However, in an experiment performed submerged culture improved oxygenation to a certain extent.

Through the statements above we conclude that Fed batch reactors seem to be the best bet. Now we move forward to the purification of cephalosporins.

Using Aspen tech or other modeling and simulation software also ensure that the cost to conduct trials will go down drastically.

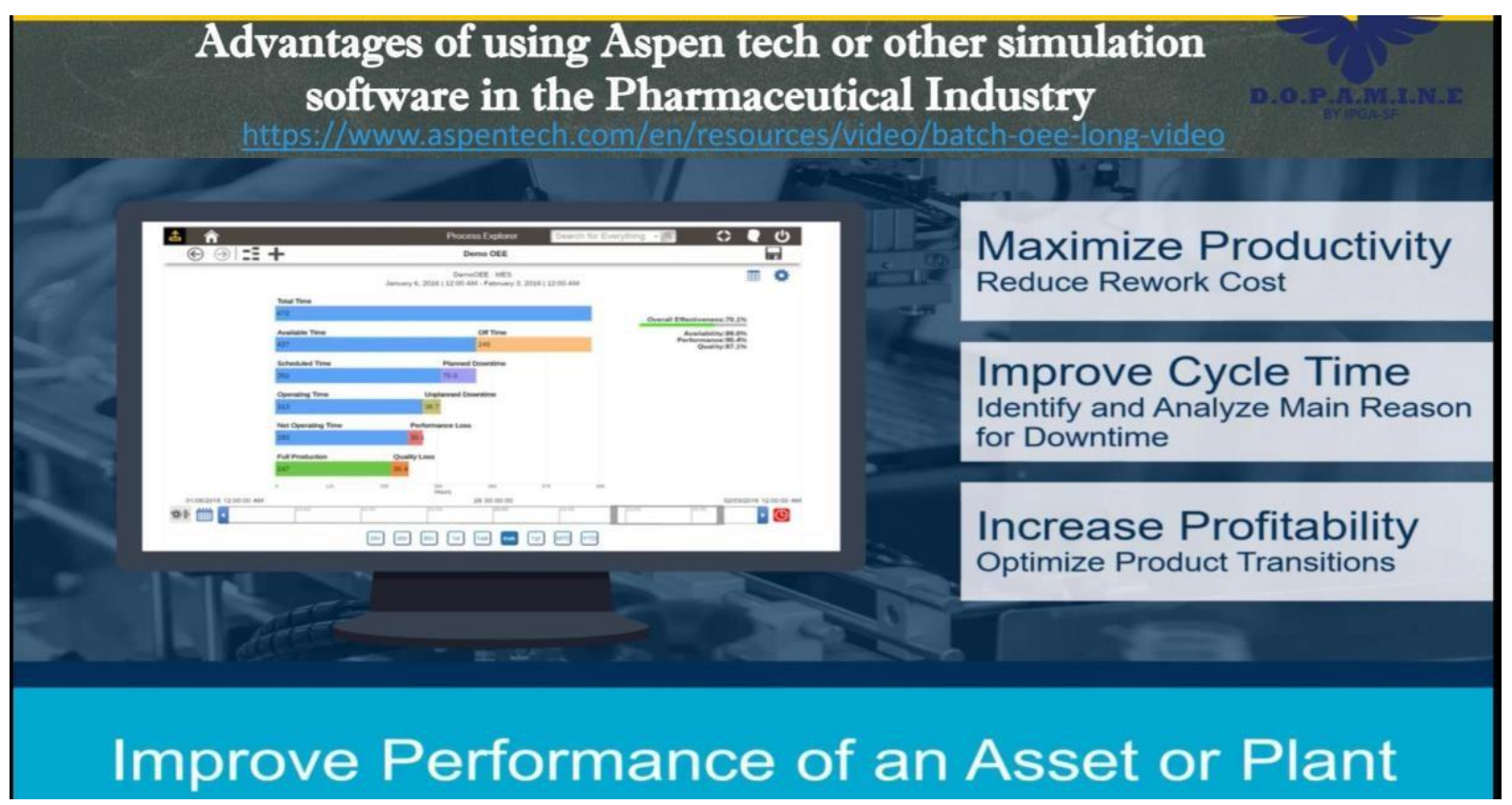

Figure 3: Aspen Tech and other similar simulation software

\subsection{Extraction of Cephalosporin C}

\subsubsection{Solid Phase Adsorption}

For cephalosporin only a few adsorbents are good due to limited capacity. Direct isolation by adsorption offers high selectivity and yield. Aromatic sorbents provide highest sorbent capacity for Cephalosporin-C (CPC).

\subsubsection{Liquid-liquid Extraction}




\subsubsection{Extraction using Lipophilic intermediates}

The problem with liquid-liquid extraction is the loss of solvent in aqueous phase because of partial miscibility with water. Also, this kind of extraction needs to be combined with a preliminary chromatographic step. In order to bypass these challenges, a lipophilic derivative of CPC is formed independent of the concentration of CPC. For extraction solvents such as ethyl acetate and methyl isobutyl ketone can be used and a purity ranging from $40-50 \%$ is achieved. Further, these derivatives can be purified by using a liquid ion exchanger.

\subsubsection{Membrane synthetic separation}

Ultrafiltration (UF), microfiltration and reverse osmosis have been studied for cell mass separation or concentration. The UF permeate is concentrated by RO and is highly purified by High performance liquid chromatography. Up to $98.5 \%$. UF is also a cost-effective technique particularly in the cross-flow mode of operation and be easily scaled up.

\subsubsection{ELM (Emulsion liquid membrane)}

For large scale operations, a counter current column operation would be preferable to a mixer settler. The device can be easily scaled up.

After having reviewed these technologies, Membrane separation technologies should be implemented further to reduce costs and gain maximum purity. ${ }^{(6)}$

\section{Issues faced in the production of Metformin}

Some of the issues with manufacturing Metformin is the time taken to set up factories. The $\%$ capacity at which India runs a factory is only $30 \%$, whereas China runs it at $70 \%$. Big time players don't want to take the risk of inventing in R\&D. However, most of the issues with Metformin deal with regulatory and infrastructural issues, which I believe doesn't cover the scope of this topic.

\section{Conclusion}

Through a series of discussions with eminent industry professionals and literature survey we concluded that fermentation-based products need require technological interventions in order to be produced here. Most of the technology used here in India isn't up to date and very little is spent on R\&D by industries, which makes overtaking China a major challenge. However, by evaluating and optimizing certain technologies we can come to a stage wherewe can retain our past glory as the Pharmaceutical giants. The conclusion of using fed batch reactors instead of only batch or continuous reactors and researching further on membrane synthetic separation for purification of cephalosporins could lead to a very cost-effective approach which is the whole aim of the paper. 


\section{References:}

1. Subir Kundu*, Ipsita Chakravarty*, Sumedha Ojha*, Kanika Kundu, Design and Development of Antibiotic Fermentation Using Different Processing Strategies: Challenges and Perspectives. Elsevier.

2. AK Srivastava, S Gupta, Fed-Batch Fermentation - Design Strategies, Elsevier.

3. Yusuf Chisti and Murray Moo-Young, Bioreactors.

4. A.J.G. Cruz!,*, A.S. Silva!, M.L.G.C, et.al, 1999. Modelling and optimization of the cephalosporin $\mathrm{C}$ production bioprocess in a fed-batch bioreactor with invert sugar as substrate. Chemical Engineering Science 54 (1999) 3137$\} 3142$.

5. Ipsita Chakravarty , Kanika Kundu , Sumedha Ojha, and Subir Kundu. 2016. Development of Various Processing Strategies for New Generation Antibiotics Using Different Modes of Bioreactors, JSM Biotechnology \& Biomedical Engineering.

6. Ghosh A.C., Mathur R.K., Dutta N.N. (1997) Extraction and purification of cephalosporin antibiotics. In: Biotreatment, Downstream Processing and Modelling. Advances in Biochemical Engineering/Biotechnology, vol 56. Springer, Berlin, Heidelberg.

\section{Acknowledgements}

We would like to extend our gratitude to IPGA Student Forum to provide the opportunity to work on this research topic under the Project DOPAMINE (EREVNA 3.0).

\section{Conflict of Interest}

The Authors declares that there is no conflict of interest. 